\title{
Die Wirkung von 6-Furfurylaminopurin auf die Atmung von Zellsuspensionen von Nicotiana tabacum
}

\author{
Von L. Bergmann \\ Aus dem Pflanzenphysiologischen Institut der Universität Göttingen \\ (Z. Naturforschg. 18 b, 942-946 [1963] ; eingegangen am 17. Januar 1963)
}

\begin{abstract}
6-Furfurylaminopurine ("kinetin") has been found to reduce markedly the respiration of cell suspensions from tissue cultures of Nicotiana tabacum var. "Samsun" in glucose containing media. The observed reduction in the oxygen consumption is not caused by an inhibition of the glucose uptake by the cells. Pyruvate, succinate, and $\alpha$-ketoglutarate can restore the respiration of cells inhibited by kinetin. The increased oxygen consumption following the addition of the acids is accompanied by an increased R.Q. It is concluded therefore, that kinetin does not inhibit the tricarboxylic acid cycle, and the suggestion is put forward that kinetin acts via the E $\mathrm{mb}$ d e n - M e y e r h of - pathway of the glucose catabolism.
\end{abstract}

In den letzten Jahren hat das als „Kinetin“ bekannt gewordene Purinderivat 6-Furfurylaminopurin in zunehmendem Maße das Interesse der Pflanzenphysiologen gefunden. Trotz der Vielzahl der Arbeiten ${ }^{1}$ jedoch, die seit der Entdeckung des Kinetins durch die Arbeitsgruppe um SкооG und MiLLER $^{2}$ erschienen sind und der Mannigfaltigkeit der beschriebenen Wirkungen auf den physiologischen Zustand der Pflanzen, wissen wir so gut wie nichts über die Angriffspunkte des Kinetins im Zellstoffwechsel.

In jüngster Zeit sind nun einige Beobachtungen mitgeteilt worden, die einen Einfluß des Kinetins oder verwandter Verbindungen auf den Kohlenhydratabbau vermuten lassen. So fanden Dedolph et al. ${ }^{3,4}$, daß das Kinetin-Analoge 6-Benzylaminopurin die Atmung von abgeschnittenen Spargeltrieben und Köpfen von Brassica oleracea var. italica herabsetzt, und Luš́ñNEC und Mitarbb. ${ }^{5}$ berichteten über eine Veränderung des $\mathrm{C}_{1} / \mathrm{C}_{6}$-Verhältnisses bei der Veratmung ${ }^{14} \mathrm{C}$-markierter Glucose durch Callusgewebe von Daucus carota nach Zugabe von 6-Benzylaminopurin. Dagegen fand GLasziou ${ }^{6}$ eine leichte Atmungssteigerung von Tabak-Markgewebe durch 6-Furfurylaminopurin in kurzfristigen Versuchen ( $3-8 \mathrm{Stdn}$.) und eine geringe Erhöhung des $\mathrm{O}_{2}$ Verbrauches in mehrtägigen Versuchen, die auf das in dieser Zeit erfolgte bessere Wachstum der Gewebe in Gegenwart von Kinetin zurückgeführt wird.

1 C. O. Miller, Annu. Rev. Plant Physiol. 12, 395 [1961].

2 C. O. Miller, F. Skoog, M. H. von Saltza u. F. M. Strong, J. Amer. chem. Soc. 77, 1392 [1955].

3 R. R. Dedolph, S. H. Wittwer u. V. Tuli, Science [New York] 134, 1075 [1961].

${ }^{4}$ G. Gilbert, Plant Physiol. 37, 509 [1962].

5 J. Luštinec, E. Petru u. V. Pokorná, Experientia [Basel] 18, 187 [1962].
Wir selbst haben im Zusammenhang mit Arbeiten über den Einfluß des Kinetins auf den Phenylpropanstoffwechsel von Gewebekulturen von Nicotiana tabacum ebenfalls die Wirkung von Kinetin auf die Atmung der Gewebe untersucht und Versuche angestellt, die beobachtete Hemmung der Glucoseveratmung näher zu analysieren. Im folgenden soll über einige Ergebnisse dieser Arbeiten berichtet werden.

Die vorliegenden Versuche wurden mit Gewebekulturen von Nicotiana tabacum var. "Samsum" durchgeführt, die 1959 als Klone aus Einzelzellen aus Wundkallusgewebe herangezogen und fortlaufend vermehrt worden sind ${ }^{7}$. Die Gewebe wurden entweder als Submerskulturen in einem flüssigen Nährmedium kultiviert $^{8}$, das Mineralsalze und Vitamine nach $W_{\text {HITE }}{ }^{9}$, $5 \%$ Cocosmilchabkochung und $10^{-7} \mathrm{~g}$ Dichlorphenoxyessigsäure/ml enthielt, oder sie wurden in der üblichen Weise auf agarhaltigen Nährböden angezogen und vor den Versuchen in Nährlösung suspendiert.

Um Fehlerquellen auszuschließen, die sich bei manometrischen Stoffwechselmessungen an Gewebeschnitten aus einer Begrenzung des Gaswechsels durch zu lange Diffusionswege sowie durch die beim Herstellen der Schnitte erfolgende Verwundung ergeben können, haben wir mit Zellsuspensionen gearbeitet, die vorwiegend aus Einzelzellen und wenigzelligen Zellgruppen bestanden (Abb. 1), und die ohne Verwundung der Gewebe erhalten werden können. Wie an anderer Stelle beschrieben ${ }^{10}$, wurden die Zellsuspensionen durch Filtration von Submerskulturen durch feine Nylonnetze unter sterilen Bedingungen gewonnen. Da die Zartheit der Zellen ein Zentrifugieren nicht erlaubte, wurden die für die Versuche notwendigen Zelldichten durch

${ }^{6}$ K. T. Glasziou, Nature [London] 179, 1083 [1957].

7 L. Bergmans, Nature [London] 184, 648 [1959].

8 L. Berganan u. G. Melchers, Z. Naturforschg. 14 b, 73 [1959].

9 P. R. White, The cultivation of animal and plant cells. The Ronald Press Comp., New York 1954.

10 L. Bergmans, J. gen. Physiol. 43, 841 [1960]. 
Abb. 1. Zellen aus einer für die manometrischen Stoffwechselmessungen benutzten Zellsuspensionen von N. tabacum (130-fach).

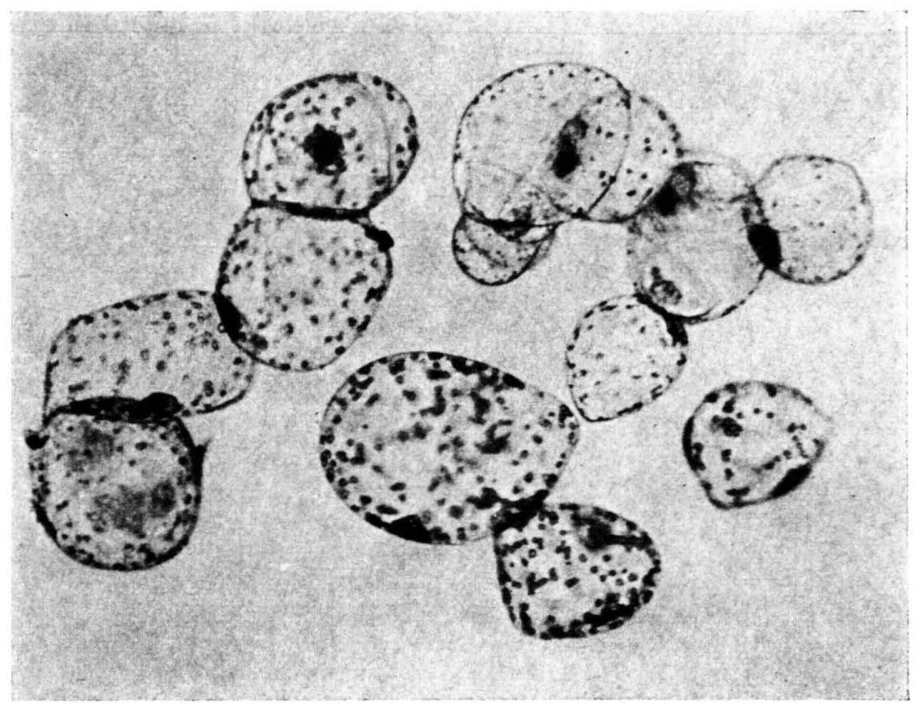

Sedimentation der Suspensionen in Meßzylindern hergestellt. Durch mehrmaliges Absitzenlassen und Aufnehmen in frischer, steriler Nährlösung wurden die Zellen dabei vor den Versuchen gewaschen. Soweit nicht anders angegeben, wurden die Zellen in einer Nährlösung suspendiert, die neben den von $\mathrm{W}_{\text {HITE }}{ }^{9}$ angegebenen Mineralsalzen und Vitaminen 2\% Glucose enthielt, und deren $p_{\mathrm{H}}-\mathrm{Wert} 5,2$ betrug.

Die Messung der Atmung erfolgte nach der einfachen „KOH-Methode“ mit 0,2 ml 20-proz. Kalilauge im Einsatz der Gefäße. Bei der Bestimmung der respiratorischen Quotienten gelangte die „ $\pm \mathrm{KOH}$-Methode“ zur Anwendung. Sämtliche Messungen wurden bei $25^{\circ} \mathrm{C}$ mit $2 \mathrm{ml}$ Zellsuspension in den Gefäßen vorgenommen. Die Zellsuspensionen enthielten in den verschiedenen Versuchen ein Frischgewicht von 100-200 mg Zellen pro $\mathrm{ml}$, was einem N-Gehalt von $0,04-0,08 \mathrm{mg} / \mathrm{ml}$ Zellsuspension entspricht.

Die verbrauchten Sauerstoffmengen wurden auf den N-Gehalt der Zellsuspensionen bezogen, der sich mit einer wesentlich größeren Genauigkeit bestimmen ließ als das Trockengewicht der Suspensionen. Die Bestimmung des N-Gehaltes erfolgte im Mikro-K j eld a h $\mathrm{l}$.

Um sicher zu gehen, daß die Zellen unter den Versuchsbedingungen in den W a r b u r g-Gefäßen nicht geschädigt werden, haben wir die Atmung einer Zellsuspension zu Beginn und am Ende einer längeren Inkubationszeit in den Gefäßen untersucht. Die Gefäße wurden hierbei am Tage $10 \mathrm{Stdn}$. mit einer Frequenz von $140 / \mathrm{min}$ und einer Amplitude von $2,5 \mathrm{~cm}$ geschüttelt und in der Zwischenzeit mit geöffneten Manometern im Apparat belassen.

Wie aus Tab. 1 hervorgeht, bleibt unter diesen Be dingungen die $\mathrm{O}_{2}$-Aufnahme der Zellen über drei Tage nahezu konstant und ist am 7. Tag um 26\% des Ausgangswertes auf $145 \mu \mathrm{l} \mathrm{O}_{2} / \mathrm{mg} \mathrm{N} / \mathrm{h}$ gestiegen. Diese Steigerung der Atmung kann als eine Folge des Wachstums der Zellen angesehen werden, denn bei der mikro-

\begin{tabular}{|c|r|c|c|c|}
\hline & 1. Tag & 2. Tag & 3. Tag & 7. Tag \\
\hline$-\mathrm{mm}^{3} 0_{2} / \mathrm{mg} \mathrm{N} \mathrm{h}$ & 115,2 & 113,8 & 117,0 & 144,9 \\
R.Q. & 1,1 & 1,12 & 1,03 & 1,05 \\
\hline
\end{tabular}

Tab. 1. Atmung einer Zellsuspension von $N$. tabacum in 2-proz. Glucosenährlösung, $p_{\mathrm{H}} 5,2$. Die angegebenen Werte sind Mittel aus je fünfstündigen Messungen.

skopischen Durchmusterung der Zellsuspensionen wurden nach Versuchsende wiederholt neugeteilte Zellen beobachtet.

Gibt man zu Beginn der Atmungsmessungen zu den Zellen Kinetin hinzu, so beobachtet man einen langsamen Abfall der $\mathrm{O}_{2}$-Aufnahme. In Tab. 2 sind die Ergebnisse einer Reihe von Versuchen zusammengestellt, in denen der Verlauf der $\mathrm{O}_{2}$-Aufnahme von Tabakzellen in Gegenwart von Kinetin in den Konzentrationen $10^{-5}, 10^{-6}$ und $10^{-7} \mathrm{~g} / \mathrm{ml}$ bestimmt wurde. Wie aus den Werten ersichtlich ist, erfolgte die Hemmung der $\mathrm{O}_{2}$-Aufnahme langsam und erreichte im Verlaufe von 24 Stdn. 50\% bzw. 30\% und $12 \%$ der Kontrollen. Der respiratorische Quotient veränderte sich dabei nicht, er zeigte mit Werten um 1 eine reine Kohlenhydratveratmung an (vgl. auch Abb. 3). Das langsame Einsetzen der Hemmung dürfte dabei vermutlich auf der langsamen Permeation des Kinetins in die Zellen beruhen.

Die Möglichkeit, daß die verminderte $\mathrm{O}_{2}$-Aufnahme auf eine Hemmung der Glucoseaufnahme durch Kinetin zurückzuführen ist, läßt sich experimentell durch Verfolgen des Einflusses des Kinetins auf die endogene Atmung prüfen. Ist nur der Auf- 


\begin{tabular}{|c|c|c|c|c|}
\hline \multirow{3}{*}{ Versuch } & $\begin{array}{l}\text { Kontrolle } \\
\text { - Kinetin }\end{array}$ & \multicolumn{3}{|c|}{$10^{-5} \mathrm{~g}$ Kinetin $/ \mathrm{ml}$} \\
\hline & $-\mathrm{mm}^{3} 0_{2} / \mathrm{mgN} / \mathrm{h}$ & \multicolumn{3}{|c|}{$0_{2}$-Verbrauch in Prozent der Kontrolle } \\
\hline & 1. -30. Stde. & 1. -5 . Stde. & 6. - 10. Stde. & 25. -29 . Stde. \\
\hline 1 & $130,0 \pm 13.5$ & 82,8 & 68,6 & 56,0 \\
\hline 2 & $113,2 \pm 14.9$ & 83,3 & 63,6 & 54,4 \\
\hline \multirow[t]{2}{*}{3} & $82,3 \pm 7.6$ & 87,5 & 79,7 & 54,4 \\
\hline & & \multicolumn{3}{|c|}{$10^{-6} \mathrm{~g}$ Kinetin $/ \mathrm{ml}$} \\
\hline 4 & $120,6 \pm 14.2$ & 88,8 & 80,1 & 71,4 \\
\hline 5 & $103,5 \pm 10.6$ & 104,8 & 86,0 & 70,9 \\
\hline \multirow[t]{2}{*}{6} & $102,6 \pm 14.4$ & 90,2 & 87,1 & 72,7 \\
\hline & & \multicolumn{3}{|c|}{$10^{-7} \mathrm{~g}$ Kinetin $/ \mathrm{ml}$} \\
\hline 7 & $85,0 \pm 7.4$ & 100,0 & 96,0 & 88,5 \\
\hline 8 & $80,0 \pm 8.6$ & 98,7 & 94,3 & 91,4 \\
\hline 9 & $87,0 \pm 12.0$ & 95,0 & 90,0 & 85,0 \\
\hline
\end{tabular}

Tab. 2. Hemmung der $\mathrm{O}_{2}$-Aufnahme von Zellsuspensionen von $N$. tabacum durch Kinetin. Die Zellen waren in einer 2-proz. Glucose-Nährlösung vom $p_{\mathrm{H}}$-Wert 5,2 suspendiert, das Kinetin wurde zu Versuchsbeginn zugegeben.

nahmemechanismus der Glucose von der Kinetineinwirkung betroffen, so sollten keine Unterschiede zwischen der endogenen Atmung von Zellen mit und ohne Kinetin auftreten. Greift dagegen das Kinetin in den Kohlenhydrat-Abbau ein, so sollte auch die endogene Atmung durch Kinetin gehemmt werden können, und die $\mathrm{O}_{2}$-Aufnahme der Zellen müßte in Gegenwart von Kinetin rascher abfallen als die der Kontrollen. Dabei muß man jedoch voraussetzen, daß die durch die erfolgende Substratabnahme in der Zelle verringerte Umsatzgeschwindigkeit nicht der allein begrenzende Faktor für die Höhe der $\mathrm{O}_{2}$ Aufnahme ist. Abb. 2 gibt einen Versuch wieder,

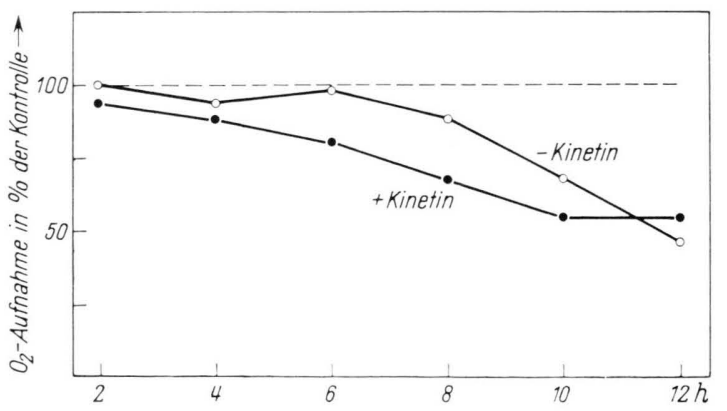

Abb. 2. Endogene Atmung einer Zellsuspension von N. tabacum in An- und Abwesenheit von Kinetin. Angabe der Werte in Prozent der $\mathrm{O}_{2}$-Aufnahme der Kontrolle mit Glucose.

der zeigt, daß diese Voraussetzung zunächst erfüllt ist. Die $\mathrm{O}_{2}$-Aufnahme der Zellen nimmt in Abwesenheit von Glucose im Medium in Gegenwart von $10^{-5} \mathrm{~g}$ Kinetin $/ \mathrm{ml}$ zunächst rascher ab als die der Kontrollen ohne Kinetin. Der nach der 8. Stde. beginnende steilere Abfall der $\mathrm{O}_{2}$-Aufnahme der Kontrolle ohne Kinetin dürfte auf die stärkere Substrat-Verarmung der Zellen zurückzuführen sein, die jetzt die Umsatzgeschwindigkeiten bestimmt. Damit ist gezeigt, daß die Herabsetzung der Glucoseatmung auf einer Einwirkung des Kinetins auf den Glucoseabbau beruhen muß.

Wir haben nun versucht, den Angriffspunkt des Kinetins auf den Glucoseabbau näher zu analysieren, indem wir die Wirkung des Kinetins auf die Veratmung von Zwischenprodukten des Kohlenhydratstoffwechsels untersucht haben. Als solche bieten sich vor allem Pyruvat und die Dicarbonsäuren des Zitronensäurezyklus an, da sie unter geeigneten Versuchsbedingungen verhältnismäßig leicht von den Zellen aufgenommen werden. Bei der Durchführung dieser Versuche wurde so vorgegangen, daß die Zellen in der glucosehaltigen Nährlösung mit Kinetin vorinkubiert wurden, bis ein Absinken der $\mathrm{O}_{2}$-Aufnahme eintrat. Daraufhin wurden aus dem seitlichen Ansatz der Gefäße $25 \mu$ Mole der betreffenden Substrate durch Kippen zugefügt, und der Gaswechsel weiter verfolgt. Die für die Versuche notwendige Erhöhung der Wasserstoffionenkonzentration wurde durch Pufferung der Lösungen mit 0,01-m. Kalium-Biphthalatpuffer erreicht.

Die Ergebnisse dieser Versuche sind in Abb. 3 zusammengestellt. Wie aus ihr ersichtlich, wird die Veratmung von Pyruvat, Succinat und $\alpha$-Ketoglutarat durch Kinetin nicht gehemmt, vielmehr läßt sich 


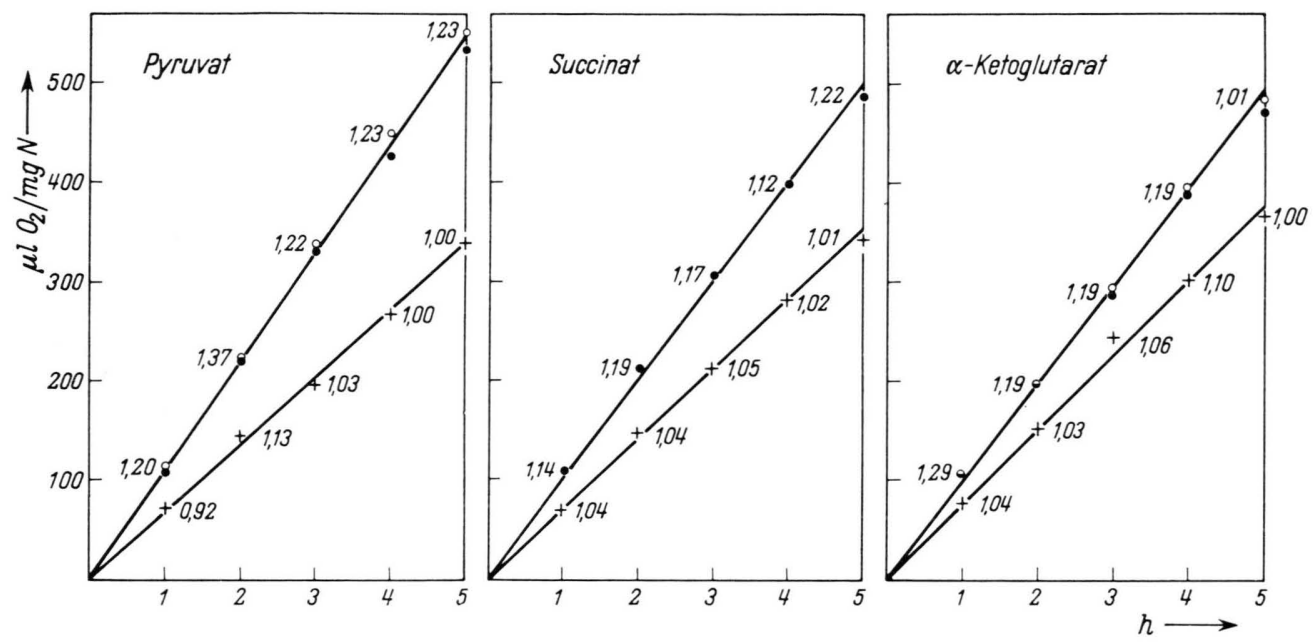

Abb. 3. Die Wirkung von Pyruvat, Succinat und $\alpha$-Ketoglutarat auf die $\mathrm{O}_{2}$-Aufnahme durch Kinetin gehemmter Zellsuspensionen von $N$. tabacum in 2-proz. Glucose-Nährlösung vom $p_{\text {H }}$-Wert 4,2. Zeiten nach Zugabe von $25 \mu$ Molen der angegebenen Substrate. +-+-+-+ Kontrolle mit $10^{-5} \mathrm{~g} / \mathrm{ml}$ Kinetin. $\bigcirc-0-0-\circ$ Kontrolle ohne Kinetin. $\bullet-\bullet-\bullet-\bullet 10^{-5} \mathrm{~g} / \mathrm{ml}$ Kinetin + angegebenes Substrat.

die durch Kinetin herabgesetzte $\mathrm{O}_{2}$-Aufnahme durch die genannten Substrate wieder auf die Höhe der Kontrollen bringen. Während die respiratorischen Quotienten der Kinetinkontrollen in den Versuchen weiter um 1 lagen, stiegen die Quotienten nach dem Zufügen der einzelnen Säuren gleichzeitig mit der $\mathrm{O}_{2}$-Aufnahme an, was darauf hinweist, daß die erhöhte $\mathrm{O}_{2}$-Aufnahme auf einer Oxydation der Säuren beruht.

Nimmt man an, daß die gebotenen Säuren in den Zellen über den Zitronensäurezyklus oxydiert werden, so folgt aus den obigen Befunden, daß die Hemmung der Glucoseveratmung durch Kinetin bei den untersuchten Geweben nicht über eine Inhibitorwirkung im Bereich des Zitronensäurezyklus oder etwa der Atmungskette erfolgt. Die Beteiligung des Zitronensäurezyklus an der Atmung der Nicotiana tabacum-Zellen wird durch die Hemmung der $\mathrm{O}_{2}$ Aufnahme mit spezifischen Inhibitoren des Zitronensäurezyklus bewiesen. Wie Tab. 3 zeigt, wird die Atmung von Zellsuspensionen aus Tabakgewebekulturen durch Malonat und Arsenit in den Konzentrationen von $0,1-m$. bzw. $0,01-m$. um rund $50 \%$ herabgesetzt.

Unsere Versuche zeigen, daß die beobachtete starke Hemmung der Glucoseatmung von Zellsuspensionen von Nicotiana tabacum durch Kinetin we-

11 R. T. Henderson, C. G. Skinner u. R. E. Eakin, Plant Physiol. 37, 552 [1962].

\begin{tabular}{|c|c|c|c|}
\hline \multirow[b]{2}{*}{ Versuchsansatz } & \multicolumn{2}{|c|}{ Sauerstoffverbrauch } & \multirow{2}{*}{$\begin{array}{c}\text { Hemmung } \\
\text { der Atmung } \\
{[\%]}\end{array}$} \\
\hline & Kontrolle & $+\underset{\text { stoff }}{\text { Hemm- }}$ & \\
\hline $\begin{array}{l}\text { 1. Malonat } 0,1-m \\
\text { 2. " } \\
\text { 3. } \\
\text { 4. Arsenit } 0,01-m \\
\text { 5. " }\end{array}$ & $\begin{array}{l}60,4 \\
72,1 \\
57,0 \\
49,8 \\
63,8\end{array}$ & $\begin{array}{l}27,6 \\
31,9 \\
28,1 \\
24,9 \\
25,8\end{array}$ & $\begin{array}{l}54,0 \\
54,5 \\
50,7 \\
50,0 \\
59,5\end{array}$ \\
\hline
\end{tabular}

Tab. 3. Die Wirkung von Malonat und Arsenit auf die Sauerstoffaufnahme von Zellsuspensionen von N. tabacum in 2-proz. Glucosenährlösung; $p_{\mathrm{H}} 5,2$. Werte in $\mathrm{mm}^{3} \mathrm{O}_{2} / 4 \mathrm{~h} / 2 \mathrm{ml}$ Suspension.

der auf einer Hemmung der Glucoseaufnahme durch die Zellen, noch einer Einwirkung auf den an der Atmung der Zellen beteiligten Zitronensäurezyklus oder die mit der Atmungskette verknüpften Enzymsysteme beruht. Man wird daher den Ansatzpunkt des Kinetins im Bereich des glykolytischen Abbauweges der Kohlenhydrate zwischen dem Glucose-6phosphat und der Brenztraubensäure suchen müssen. Dabei wäre an die Möglichkeit der Hemmung spezieller Enzymsysteme zu denken oder ${ }^{-}$an eine Störung der für die Verarbeitung der Kohlenhydrate notwendigen Phosphorylierungen. Der Gedanke einer Interferenz des Kinetins mit Phosphorylierungs-Vorgängen wird dabei durch die chemische Verwandtschaft des Kinetins mit den an den Phosphorylierungs-Schritten beteiligten Purinkörpern nahegelegt, während die Möglichkeit einer spezifischen Hemmung von Enzymsystemen auf Grund der 
Befunde von Henderson, Skinner und Eakin ${ }^{11}$ über die Hemmung der Xanthinoxydase durch Kinetin nicht ausgeschlossen erscheint.

Die in unseren Versuchen gefundene Hemmung der Glucoseveratmung durch Kinetin scheint in einem gewissen Gegensatz zu der fördernden Wirkung des Kinetins auf das Wachstum von GewebeKulturen ${ }^{1}$ zu stehen. Dieser Gegensatz erklärt sich zunächst aus der unterschiedlichen Höhe der verwandten Dosen. Während Kinetin-Konzentrationen von $10^{-8}$ bis $10^{-7} \mathrm{~g} / \mathrm{ml}$ auch bei unseren Geweben das Wachstum deutlich fördern, wird es durch Konzentrationen von $10^{-6}$ und $10^{-5} \mathrm{~g} / \mathrm{ml}$ gehemmt. Die Anwendung der hohen unphysiologischen Dosen war in der vorliegenden Arbeit jedoch notwendig, um den Einfluß des Kinetins auf die Atmung klar herauszuarbeiten. Wie aus Tab. 2 hervorgeht, wird die $\mathrm{O}_{2}$-Aufnahme der Zellen auch durch $10^{-7} \mathrm{~g} / \mathrm{ml}$ gehemmt, doch läßt sich die geringfügig herabgesetzte $\mathrm{O}_{2}$-Aufnahme unter den vorliegenden Versuchsbedingungen mit einem $P$ zwischen 0,02 und 0,05 nach P̈̈тAU ${ }^{12}$ nicht einwandfrei sichern, so daß die Wirkung höherer Konzentrationen zum Beweis der Signifikanz der mit $10^{-7} \mathrm{~g} / \mathrm{ml}$ beobachteten Hemmung herangezogen wurde.

Während man für die Wachstumshemmung durch höhere Kinetin-Konzentrationen den stark herabgesetzten Kohlenhydratumsatz der Zellen verantwortlich machen kann, der schließlich zu irreparablen Schädigungen führt, bleibt für schwächere Kinetindosen der Gegensatz zwischen der im kurzfristigen Versuch festgestellten Hemmung der Glucoseverat-

12 K. P̈̈тач, Biol. Zbl. 63, 152 [1943].

13 I. A. Sargent u. F. Skoog, Plant Physiol. 35, 934 [1960].

14 L. Bergman, unveröffentl. Ergebnisse. mung und der in zeitlich längeren Experimenten beobachteten Wachstumsförderung zunächst noch zu klären. Man könnte hierzu annehmen, daß die durch geringe Kinetin-Konzentrationen gehemmte Glucoseveratmung bei längerer Kultur der Zellen wieder auf das Normalmaß heraufreguliert wird, indem ein vermehrter Anteil der Glucose über den Pentose-Phosphat-Zyklus verarbeitet wird, so daß es nicht zu einer Wachstumshemmung auf Grund eines verminderten Kohlenhydratumsatzes kommt. Für eine derartige vermehrte Beteiligung des Pentosephosphatzyklus am Kohlenhydratabbau sprechen u. a. Befunde über einen in Gegenwart von Kinetin erhöhten Phenylpropanspiegel in Gewebekulturen. So berichten SARGENT und SKOOG ${ }^{13}$ über eine starke Vermehrung von Scopolin und Scopoletin in Tabakgeweben unter dem Einfluß von Kinetin, während wir selbst eine erhöhte Synthese von Lignin und verschiedenen anderen Phenylpropan-Derivaten in Gegenwart von Kinetin beobachteten ${ }^{14}$. Da die Bil. dung der Phenylpropane nach unserem heutigen Wissen aus den Intermediärprodukten des Pentosephosphat-Zyklus erfolgt ${ }^{15,16}$, kann man in ihrem vermehrten Auftreten einen Hinweis auf die in den Geweben durch Kinetin bewirkte Umsteuerung des Kohlenhydrat-Stoffwechsels sehen. Versuche zur weiteren Klärung dieser Frage sind zur Zeit im Gange.

Frau Dagmar Micke danke ich für sorgfältige Mitarbeit bei der Durchführung der Versuche, der D e u t . schen Forschungsgemeinschaft für die großzügige Unterstützung der Arbeit.

15 A. C. Neish, Annu. Rev. Plant Physiol. 11, 55 [1960].

16 H. Rezvik, Ergebn. Biol. 23, 14 [1960]. 